\title{
ANÁLISIS INTEGRAL DE LA GESTIÓN DE LA CONVIVENCIA ESCOLAR. UNA PROPUESTA DE EVALUACIÓN
}

\author{
COMPREHENSIVE ANALYSIS OF THE MANAGEMENT OF SCHOOL COEXISTENCE. A \\ PROPOSAL EVALUATION
}

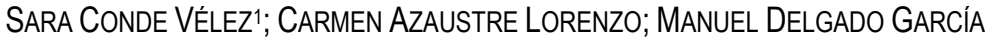 \\ Universidad de Huelva (España)
}

\section{RESUMEN}

Ante la certeza del carácter integral que caracteriza el abordaje de la convivencia en centros educativos, y la ausencia de estudios que realicen un tratamiento complejo, este estudio se propone analizar la estructura global de la gestión de la convivencia en centros de educación secundaria. Para ello, toma como marco de referencia el modelo de la European Foundation Quality Management (EFQM en adelante) para la evaluación de la Calidad. La muestra es intencional y la configura un total de 46 centros educativos de Andalucía que pertenecen a la red "Escuela: Espacio de Paz", y con una experiencia en la misma superior a los ocho años. Se utiliza el cuestionario validado a través de una revisión teórica, de los componentes del modelo EFQM y del juicio de expertos, que se aplica a 46 coordinadores y 46 familiares integrantes de las comisiones de convivencia escolar. Para el análisis se realiza una reducción de variables a través del análisis factorial, que concluye con un análisis de ecuaciones estructurales que permite observar las relaciones de influencia entre factores. Los resultados del estudio, con el ánimo de estimular la aplicación de otros modelos alternativos o complementarios, confirman la validez del modelo EFQM para el análisis integral la convivencia escolar, si bien, se insiste en la necesidad de replicar el estudio en otros contextos y de fortalecer la validez teórica del modelo.

Palabras clave: Gestión de la convivencia; Convivencia escolar; Desarrollo organizativo; Propuesta de evaluación; Modelo EFQM.

\section{ABSTRACT}

Faced with the certainty of completeness that characterizes the approach of coexistence in schools, and the lack of studies undertaken a complex treatment, this study aims to analyze the overall structure of the management of coexistence in secondary education. To do this, it takes as a reference the model of the European Foundation for Quality Management (EFQM on) for the evaluation of quality. The sample is intentional and set a total of 46 schools in Andalusia that belong to the "School: Space for Peace" network, and with an experience in the same over eight years. The questionnaire validated by a theoretical review of the components of the EFQM model and expert opinion that 46 coordinators and 46 family members of school life commissions apply is used. For analysis variables reduction is done through factor analysis, which concludes with an analysis of structural equations that allows us to observe the relationships between factors of influence. The results of the study, with the aim of stimulating the use of altemative or complementary models, confirm the validity of the EFQM model for the comprehensive analysis of school life, although it stresses the need to replicate the study in other contexts and strengthen the theoretical validity of the model.

Key words: Management of community life at school; Community life at school; Organizational development; Proposal evaluation; Model EFQM.

${ }^{1}$ Corresponding author: Sara Conde Vélez. E -mail: sara.conde@dedu.uhu.es 


\section{Introducción}

Es reconocido por la comunidad científica, que la gestión de la convivencia escolar es un proceso complejo, multidimensional y global, en el que intervienen e interactúan múltiples factores de carácter social, psicológico, y educativo (Calvo, Leal y Roldan, 2006; Olweus, 1993 y Pedrero, 2011). En este sentido, se pueden identificar investigaciones que se dedican al estudio de factores específicos que tienen influencia directa sobre la convivencia. A modo de ejemplo, los estudios de Eskildsen y Dahlgaard (2000) y Flynn et al. (1994), demuestran la correlación positiva del liderazgo, factor que es puesto de relevancia a través de estudios como el de Campo, Fernández y Grisaleña, (2005) con otros factores como la gestión de los recursos, la programación y otras variables que favorecen un clima de convivencia positivo. Aportaciones como la de Tuvilla (2004) que ponen en valor el sentido de comunidad mostrando la influencia de la motivación del profesorado sobre el liderazgo educativo y el estilo de gestión. Otros estudios han demostrado el poder de los procesos como materialización de los planes y recursos, como vínculo entre insumos, la planificación y los resultados (Kanji y Tambi, 1999). Al margen de estudios empíricos, se encuentran multitud de postulados, opiniones de expertos, sugerencias, incluso instrucciones y decretos en el ámbito de diversos gobiernos territoriales que ponen de relieve la importancia de la elaboración democrática de las normas (Caballero, 2010; Acosta, 2003; Cidad, 1996); las actividades dirigidas a favorecer la participación de las familias y el centro educativo (Caballero, 2010; Cascón, 2000; Esperanza, 2006); las actividades que se realizan en las llamadas "aulas de convivencia" (Esperanza, 2006); la atención a la diversidad y el aprendizaje significativo, la gestión de los compromisos de convivencia y protocolos a seguir en la mediación pacifica de los conflictos (Esperanza, 2006; Díaz- Aguado, 2006; Torrego et al, 2000; Uranga, 1998; Torrego, 2003 y Otero, 2001).

Sin embargo, dada la dificultad y complejidad del fenómeno, existe una ausencia de estudios empíricos que aborden de forma integral el análisis de la gestión de la convivencia. Son escasos los trabajos que plantean un marco de planificación y evaluación riguroso respecto a lo que los propios centros están desarrollando (Fernández-García, 2008; García-Raga y López-Martín, 2011).

Este estudio se plantea como reto y objetivo realizar un análisis integral que permita corroborar las sugerencias, propuestas y postulados sobre el tratamiento de la convivencia desde los centros educativos. Conscientes de que se tratará de un acercamiento estructural y global al problema con base empírica y susceptible de profundización por múltiples vías metodológicas y dimensiones que permitan ir construyendo un marco teórico válido.

Desde una perspectiva metodológica en la evaluación de la convivencia escolar se han empleado diferentes instrumentos no estandarizados (Fernández-Baena et al., 2011; Fernández-García, 2008; Ortega, et al., 2009), lo que cuestiona la fiabilidad y validez de las investigaciones además de dificultarse la comparación de los resultados y de las aplicaciones de cada uno de ellos.

Es por ello, que para abordar tal objetivo, se toma de referencia el modelo de la European Foundation Quality Management (modelo EFQM, en adelante) por los siguientes motivos: a) se trata de un esquema genérico para la gestión de la calidad; b) es concebido como un marco multidimensional, adaptable y flexible en su materialización; y c) no contradice los hallazgos encontrados por la investigación empírica sobre la gestión de la convivencia escolar.

Asimismo, son muchas las autoridades académicas y políticas que han destacado las bondades de la aplicación del modelo a la educación. Mel Farrar (2000) señala que el modelo puede ser usado beneficiosamente en una organización educativa con una estructura complicada pero con claridad de visión y objetivos, identificando e implicando a los clientes, a los empleados, con una cultura de evaluación continua y una guía para la mejora continua. Mateo (2000) en defensa de este modelo argumenta que ya ha sido adaptado a la realidad educativa y que resulta útil para la reflexión interna de los centros y el desarrollo organizativo.

La Comisión Europea, promueve, desde 1997, la utilización del Modelo EFQM para la evaluación de instituciones educativas, y son muchas las administraciones educativas públicas que lo han adaptado. En el sector educativo, la Agencia Andaluza de Evaluación Educativa (AGAEVE) promueve entre sus líneas de actuación sobre modelos de autoevaluación de centros, programas y servicios educativos la elaboración de guías de autoevaluación de centros y servicios educativos conforme a los estándares del modelo de la EFQM. 
Empíricamente, resultado de trabajos como los de Biehl (2000), Kanji et al (1999), Montano y Glenn (1999), Spanbauer (1995) y Weller (2000), se extraen las siguientes conclusiones. En primer lugar, la viabilidad de la utilización de gestión de la calidad en el contexto educativo. En segundo lugar, la eficacia de este enfoque de gestión para la mejora de las instituciones educativas en áreas tales como planificación, recursos humanos, administración de recursos y gestión de procesos educativos y administrativos. Por otro lado Saraiva, Rosa y d'Oreyi (2003) en una investigación desarrollada sobre la incidencia del modelo en una muestra de 47 escuelas Portuguesas, concluyen diciendo que el modelo de la EFQM es fácil de aplicar y adaptarse en la política de mejora de las escuelas.

\section{Descripción de los elementos del modelo}

El modelo EFQM de Excelencia es un marco de trabajo no-prescriptivo que reconoce que la excelencia de una organización se puede lograr de manera sostenida mediante distintos enfoques. Dentro de este marco general existen una serie de factores claves que constituyen la base del modelo:

- Liderazgo. La literatura identifica el "liderazgo" como uno de los factores críticos de éxito para mantener la mejora continua en cualquier organización (Zairi, 1994; Taffinder, 1995). En la investigación realizada por Campo, Fernández y Grisaleña (2005), uno de los aspectos destacados para la mejora de la convivencia, hace referencia al liderazgo, concretamente a la presencia de un coordinador de proyectos capaz de liderar estos procesos.

- Planificación. Política y estrategia deben ser puestos en práctica a través de la implantación de los procesos claves, la política adecuada y la gestión del personal y a través de la creación de asociaciones (Winn y Cameron, 1998). En este sentido, otro de los aspectos que funciona en la mejora de la convivencia de los centros estudiados por Campo, Fernández y Grisaleña (2005) es documentar la realidad, es decir, la escuela y la comunidad escolar deben unirse en un diagnóstico compartido de sus puntos fuertes y débiles en el ámbito de la convivencia.

- Gestión del personal. En el campo de la educación, Detert y Jenni (2000) y Osseo-Asare y Longbottom (2002) destacan el papel de la formación como factor clave y subrayan la adquisición continua de nuevos conocimientos y habilidades de todos los empleados.

- Recursos. La importancia de las relaciones con los proveedores y la gestión de los recursos tangibles e intangibles es un aspecto abordado con frecuencia en la literatura sobre gestión de calidad (Eskildsen y Dahlgaard, 2000). Eskildsen y Dahlgaard (2000) en un análisis empírico del modelo EFQM, descubrió una relación positiva significativa entre la gestión de alianzas y gestión de procesos.

En este sentido Díaz-Aguado (2002) en un estudio realizado sobre las condiciones que mejoran la convivencia escolar, habla de poner a disposición del profesorado los medios que permitan desarrollar una convivencia democrática. Asimismo, continúa diciendo que todas las innovaciones necesarias para educar la ciudadanía democrática exigen contar con condiciones que permitan a los profesores llevarlas a cabo.

- Procesos. El análisis de la estructura de la relaciones en el modelo EFQM, la gestión de procesos parece ser el vínculo entre los otros agentes y los resultados. Los procesos claves se consideran aquellos que tienen un efecto significativo sobre los resultados críticos para una organización determinada (Kanji y Tambi, 1999).

- Enseñar convivencia no es un tema sino una práctica, una lección viva diaria; por ello, el único camino es la cooperación, el dialogo y la confrontación crítica y respetuosa de ideas (Ortega y Del Rey, 2006). Diaz- Aguado (2006) propone una serie de actividades a la hora de mejorar la convivencia como son: Discusiones y debates entre alumnos, experiencias de solidaridad y responsabilidad, experiencia para resolver los conflictos desarrollando la comunicación, mediación, negociación y la reflexión así como experiencias que les ayuden a tomar decisiones de forma democrática. 
- Resultados en los clientes. Pretende conocer qué logros está alcanzando la organización en relación con sus clientes externos (EFQM, 2003).

Esta idea trasladada al ámbito de la convivencia, se materializa en las relaciones externas, las prácticas que el profesorado establece con las familias, cuando éstas tratan de ejercer algún tipo de control (que en no pocas ocasiones son interpretados como una injerencia inadmisible) son las que más conflictos crean en los centros (Bardisa, 2001).

- Resultados en el personal. Hace referencia a los logros alcanzados por la organización en relación con las personas que la integran (EFQM, 2003). En el caso de la convivencia escolar y de acuerdo con el Informe Estatal sobre Convivencia Escolar (2010) un importante indicador de la calidad de la vida del profesorado y de su disponibilidad para construir la convivencia es su sentido de realización personal.

- Resultados en la sociedad. Se refiere a los logros que está alcanzando la organización en la sociedad, a nivel local, nacional e internacional (según resulte pertinente) (EFQM, 2003). Trabajar desde la comunidad misma para resolución de conflictos es la clave para incidir en muchas situaciones conflictivas que tienen su raíz en el contexto social del cual proviene el alumnado que acude a los centros de enseñanza (Cuevas, 2006)

- Resultados claves de la organización. Se refiere a los logros que está alcanzando la organización con relación al rendimiento planificado (EFQM, 2003). De acuerdo con los resultados obtenidos en el Estudio Estatal sobre Convivencia Escolar (2010) mejorar la convivencia es una condición necesaria aunque no suficiente para reducir el fracaso escolar, y la convivencia se construye desde cada actividad que tiene lugar en la escuela, incluida la enseñanza de las materias.

\section{Objetivos}

El objetivo de este estudio consiste en validar el modelo EFQM, sin ánimo de excluir otros modelos, para el análisis integral de la gestión de la convivencia escolar en los centros educativos.

De modo más específico, los objetivos de esta investigación son los siguientes:

- Identificar los criterios, en términos del modelo EFQM, que permitan una descripción global y comprensiva de la gestión de la convivencia de los centros educativos de educación secundaria.

- Comprobar la relación entre los criterios, contenidos en el modelo EFQM, que permita una comprensión global de la gestión de la convivencia escolar, y sus efectos sobre los resultados.

\section{Hipótesis}

Atendiendo al objetivo general de esta investigación se plantea la siguiente hipótesis: El modelo EFQM es una herramienta válida para analizar la convivencia escolar desde una perspectiva integral y global.

A continuación, se muestra el modelo teórico, amparado en los planteamientos precedentes, que se somete a contraste empírico (ver Figura 1): 


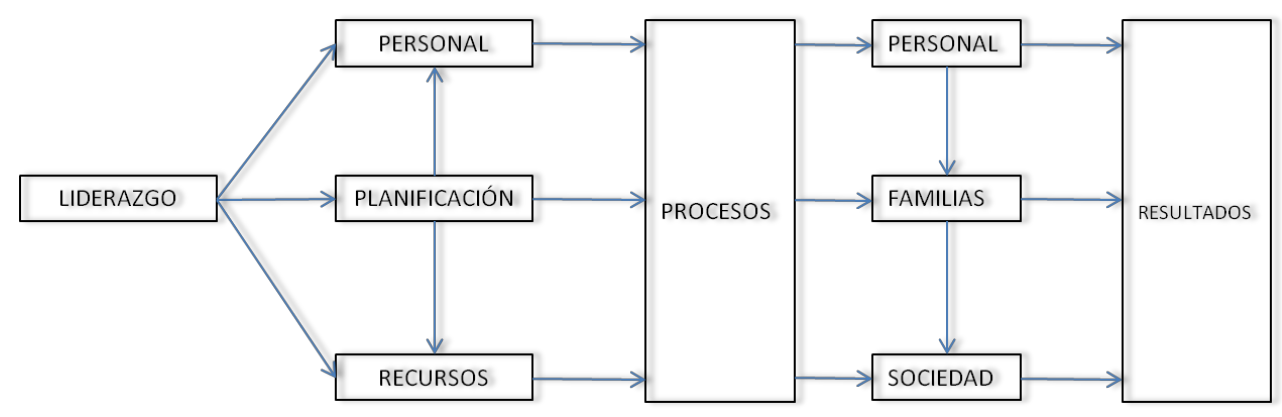

Figura 1. Hipótesis sometida a contraste

Por lo tanto, según el modelo teórico que se presenta, las hipótesis que se plantean son las siguientes:

- El Liderazgo actúa como fuerza motriz en la gestión de la convivencia escolar.

- La Planificación influye, sirviendo de referencia, en la gestión del Personal, los Recursos y el desarrollo de los Procesos.

- Los Procesos actúan de vínculo entre los factores Agentes y los resultados.

- El modelo tiene carácter sistémico y considera a los agentes en su conjunto.

\section{Método}

\subsection{Muestra}

Para la selección de la muestra, se ha utilizado un muestreo intencional adoptando la antigüedad y experiencia como criterio objetivo. Concretamente la muestra se compone de 46 centros de educación secundaria de Andalucía integrados en la red «Escuela: Espacio de Paz» durante un periodo superior a 8 años".

Para la recogida de datos se optó por tomar como informantes a 46 profesores y 46 familiares que participan en las Comisiones de Convivencia de los centros de Educación Secundaria seleccionados. Para obtener una medida única por centro, las comisiones de convivencia aplicaron el método de medida interjuez, estableciendo un acuerdo entre los informantes sobre el acontecimiento que se estaba evaluando.

\subsection{Procedimiento, Instrumento y Variables}

El instrumento utilizado para la recogida de los datos es el cuestionario, basado en la estructura del modelo EFQM, dada su rasgo multidimensional y versatilidad, tomando sus dimensiones como factores modelados sobre la base de los hallazgos empíricos, postulados y medidas institucionales comúnmente aceptadas y consolidadas (Orden de 20 de junio de 2011, Decreto 19/2007, de 23 de enero).

El cuestionario se estructura respetando las dimensiones del modelo (ver Anexo). A saber: liderazgo, planificación, gestión del personal, recursos, procesos, satisfacción de los familiares, satisfacción del personal, impacto en la sociedad y resultados en el centro. Cada una de estas dimensiones queda integrada por una serie de indicadores a valorar a través de una escala tipo likert con valores comprendidos entre 1 y 4, siendo 1 nada, 2 algo, 3 bastante y 4 mucho, incluyendo también la opción no sabe/no contesta. Estos valores hacen referencia a la frecuencia con la que se realizan cada una de las acciones propuestas a modo de indicadores.

En el proceso de validación teórica seguido se toman dos medidas:

- La adaptación del modelo a la gestión de la convivencia escolar. Respetando el significado de las dimensiones del modelo se adaptan a la base epistemológica, normativa y postulados aceptados 
sobre aspectos de gestión o factores que influyen en la materialización de la convivencia en los centros educativos.

- Juicios de Expertos. Se procedió a la revisión del cuestionario, por parte del grupo de expertos, valorando la pertinencia de los indicadores de convivencia en cada una de las dimensiones del modelo.

La fiabilidad del instrumento se analiza a través de dos procedimientos, Alfa de Cronbach y procedimiento de dos mitades. En este sentido, aplicado el test de Alfa de Cronbach para 173 variables en una muestra de 46 centros, se obtuvo un índice de fiabilidad de .972. Asimismo, también se aplicó el test a cada una de las dimensiones, obteniendo los resultados que aparecen en la Tabla 1.

Tabla 1. Resultados del Test de Alfa para cada una de las dimensiones del cuestionario

\begin{tabular}{|l|l|}
\hline Dimensiones & Alfa de Cronbach \\
\hline Liderazgo & .861 para 31 elementos \\
\hline Planificación & .871 para 33 elementos \\
\hline Gestión del Personal & .80 para 10 elementos \\
\hline Gestión de los Recursos & .844 para 21 elementos \\
\hline Procesos & .955 para 35 elementos \\
\hline Satisfacción de los familiares & .897 para 18 elementos \\
\hline Satisfacción del personal & .918 para 24 elementos \\
\hline Impacto en la sociedad & .734 para 5 elementos \\
\hline Resultados obtenidos en el centro & .898 para 14 elementos \\
\hline
\end{tabular}

La aplicación de la prueba de dos mitades al cuestionario completo ofreció como resultado .954. Asimismo, se aplicó a cada una de las dimensiones con los resultados recogidos en la Tabla 2.

Tabla 2 Resultados del Test de dos mitades para cada una de las dimensiones del cuestionario

\begin{tabular}{|l|l|}
\hline Dimensiones & Dos Mitades \\
\hline Liderazgo & .917 para 31 elementos. \\
\hline Planificación & .73 para 33 elementos \\
\hline Gestión del Personal & .844 para 10 elementos \\
\hline Gestión de los Recursos & .938 para 21 elementos \\
\hline Procesos & .958 para 35 elementos \\
\hline Satisfacción de los familiares & .817 para 18 elementos \\
\hline Satisfacción del personal & .88 para 24 elementos \\
\hline Impacto en la sociedad & .58 para 5 elementos \\
\hline Resultados obtenidos en el centro & .843 para 14 elementos \\
\hline
\end{tabular}

Los resultados obtenidos en el Alfa de Cronbach sitúan a todas las dimensiones, cuanto menos, en valores aceptables. Es en el procedimiento de las dos mitades, donde la dimensión "Impacto en la sociedad", ofrece un valor pobre (.58 para 5 elementos). Se encuentra la explicación en el escaso número 
de elemento que compone dicha dimensión, ya que este procedimiento consiste en hallar la correlación entre dos mitades paralelas del test, en este caso de la dimensión.

Por lo tanto se decide mantener esta dimensión ya que en el Alfa de Cronbach sí obtiene un valor aceptable.

\subsection{Análisis de datos}

Para la confirmación de las hipótesis planteadas se utilizó la modelización de ecuaciones estructurales. Esta técnica se basa en que toda teoría implica un conjunto de correlaciones, y si tal teoría es válida debe ser posible reproducir los patrones de correlación (supuestos) en datos empíricos.

Una vez corroboradas las condiciones que permiten la aplicación de esta técnica, entre ellas los altos valores de correlación del test de Pearson, se procede al análisis factorial, con el objeto de reducir el volumen de variables implicadas, concentrando las variables en factores y de esta forma facilitar la confirmación del modelo EFQM como marco válido para una comprensión integral del abordaje de la convivencia escolar.

El programa utilizado para el modelaje es Amos 18.0.

\section{Resultados}

\subsection{Reducción factorial previa}

Se hace un análisis factorial exploratorio con la intención de concentrar la pertenencia de los ítems a un factor y así discriminar mejor entre factores. Se recurre a una reducción factorial aplicando una rotación ortogonal con el método Quartimax, el objetivo es que cada variable tenga correlaciones elevadas con un pequeño número de factores. Para ello, maximiza la varianza de las cargas factoriales al cuadrado de cada variable en los factores. Con ello, se logra que cada variable concentre su pertenencia en un determinado factor, esto es, presente una carga factorial alta mientras que, en los demás factores, sus cargas factoriales tienden a ser bajas, quedando más evidente hacia qué factor se inclina con más fuerza cada variable. Los resultados son los siguientes respecto a cada una de las dimensiones consideradas:

- Liderazgo. Se identifican cuatro factores que llegan a explicar el $72.50 \%$ de la varianza del conjunto de las variables. A saber: a) F1.1. Conducción y gobierno del Plan de convivencia (38.77\%); b) F1.2. Compromiso con la mejora de la convivencia escolar (14.86\%); c) F1.3. Responsabilidad del Equipo Directivo (10.49\%); d) F1.4. Coordinación en la elaboración del Plan de Convivencia (8.36\%).

- Planificación. Se identifican nueve factores que saturan el $77.53 \%$ de la varianza del conjunto de las variables. A saber: a) F2.1. Análisis de la realidad del centro (22.86\%); b) F2.2. Planificación del aula de convivencia (14.95\%); c) F2.3. Planificación de los compromisos de convivencia (9.44\%); d) F2.4. Características de las familias y cooperación (6.57\%); e) F2.5. Información y sensibilización (5.89\%); f) F2.6. Análisis de las prácticas educativas $(5.28 \%)$; g) F2.7. Características del personal del centro (4.73\%); h) F2.8. Conocimiento y propuesta del Plan (4.15\%); i) F2.9. Características del centro y Plan de convivencia (3.62\%).

- Personal. Se identifican tres factores que saturan el $79.10 \%$ de la varianza del conjunto de las variables. A saber: a) F3.1. Formación del Personal (36.97\%); b) F3.2. Funciones y tareas del Educador/a Social (25.35\%); c) F3.3. Plan de trabajo de la comisión de convivencia (16.77\%).

- Recursos. Se identifican cinco factores que llegan a explicar el $74.79 \%$ de la varianza del conjunto de las variables. A saber: a) F4.1. Aula de convivencia (33.45\%); b) F4.2. Instrumentos y recursos $(21.60 \%)$; c) F4.3. Profesorado de apoyo específico $(8.40 \%)$; d) F4.4. Espacios alternativos (5.92\%); e) F4.5. El Educador/a Social (5.40\%).

- Procesos. Se identifican tres factores que llegan a explicar el $60.11 \%$ de la varianza del conjunto de las variables. A saber: a) F5.1. Utilidad de las acciones propuestas para la mejora de la 
convivencia escolar (41.76\%); b) F5.2. Facilitar la integración del alumnado (10.73\%); c) F5.3. Participación de la comunidad educativa $(7.60 \%)$.

- Satisfacción Familias. Se identifican tres factores que llegan a explicar el $57.63 \%$ de la varianza del conjunto de las variables. A saber: a) F6.1. Satisfacción respecto al plan de convivencia (38.76\%); b) F6.2Satisfacción respecto a las quejas y resultados del plan (10.61\%); c) F6.3. Satisfacción entrevistas (8.25\%).

- Satisfacción Personal. Se identifican cuatro factores que saturan la $59.50 \%$ de la varianza del conjunto de las variables. A saber: a) F7.1. Satisfacción respecto al Plan de convivencia (36.77\%);

b) F7.2. Participación del personal en las mejoras del Plan (10.82\%); c) F7.3. Motivación (6.98\%);

d) F7.4. Aula de convivencia (4.91\%).

- Impacto en la Sociedad. Se identifica un único factor que explica el $100 \%$ de la varianza del conjunto de las variables. F8.1. Satisfacción respecto a los efectos en la comunidad (100\%).

- Resultados del Plan. Se identifican cuatro factores que llegan a explicar el $71.92 \%$ de la varianza del conjunto de las variables. A saber: a) F9.1. Conductas contrarias y gravemente perjudiciales (45.88\%); b) F9.2. Favorecer las relaciones entre grupos culturales (10.31\%); c) F9.3. Reducción de faltas injustificadas (8.22\%); d) F9.4. Reducir actos de disrupción en el aula (7.49\%).

\subsection{Confirmación del modelo}

Los índices de bondad de ajuste indican que el modelo (ver Figura 1) se ajustó bien a los datos (Chisquare/df= 1.65; $p>.01 ; \mathrm{CFI}=.930 ; \mathrm{IFI}=.935 ; \mathrm{NFI}=.851 ; \mathrm{TLI}=.873)$.

El modelo muestra un efecto directo del factor Liderazgo sobre el factor Gestión del Personal ( $\beta=.15$, $p=.358$ ) y sobre el factor Planificación ( $\beta=.59, p<.001)$. Asimismo, el factor liderazgo tiene una influencia inversa sobre el factor Recursos $(\beta=-.10, p<.532)$, el sentido negativo de su influencia, se identifica con la gestión del «aula de convivencia». De lo que podría suponerse que altos valores de Liderazgo no hacen necesario el concepto convencional del recurso denominado «aula de convivencia».

Tanto la influencia del factor Liderazgo sumada a la del factor Planificación, explican el $22 \%$ de la varianza del factor Gestión del Personal. Asimismo, es únicamente la influencia del factor Liderazgo ( $\beta=.59$, $p<.001$ ) la que explica el $35 \%$ de su varianza.

Por otro lado, el factor Planificación, influye directamente sobre el factor Gestión del personal ( $\beta=.36$, $p=.028)$ y sobre el factor Recursos $(\beta=.60, p<.001)$.

Respecto al factor Procesos, se comprueba que el $51 \%$ de su varianza es explicada por los siguientes factores:

- El factor Gestión del personal ( $\beta=.24, p=.043)$.

- El factor Planificación $(\beta=.38, p=.005)$.

- El factor Recursos $(\beta=.28, p=.026)$.

En cuanto a los factores resultados se puede verificar el efecto del factor Procesos sobre los mismos, en la siguiente medida:

- El $44 \%$ de la varianza del factor Satisfacción Familiar es explicado directamente por el factor Procesos ( $\beta=.31, p=.081$ ) y por el factor Satisfacción del Personal $(\beta=.40, p<.001)$.

- El $60 \%$ de la varianza del factor Satisfacción del Personal $(60 \%)$ es explicada directamente por el factor Procesos ( $\beta=.77, p<.001)$.

- El $11 \%$ de la varianza del factor Impacto en la Sociedad es explicada directamente por el factor Procesos $(\beta=.10, p=.557)$ y por el factor Satisfacción Familiar ( $\beta=.26, p=.146)$. 
Finalmente, se encuentra en el modelo que el $52 \%$ de la varianza del factor Resultados obtenidos por el Centro, es explicada por los siguientes factores:

- Satisfacción del personal $(\beta=.58, \beta<.001)$.

- Satisfacción de las familias $(\beta=.02, p=.911)$.

- Impacto en la sociedad ( $\beta=.30, p=.007)$.

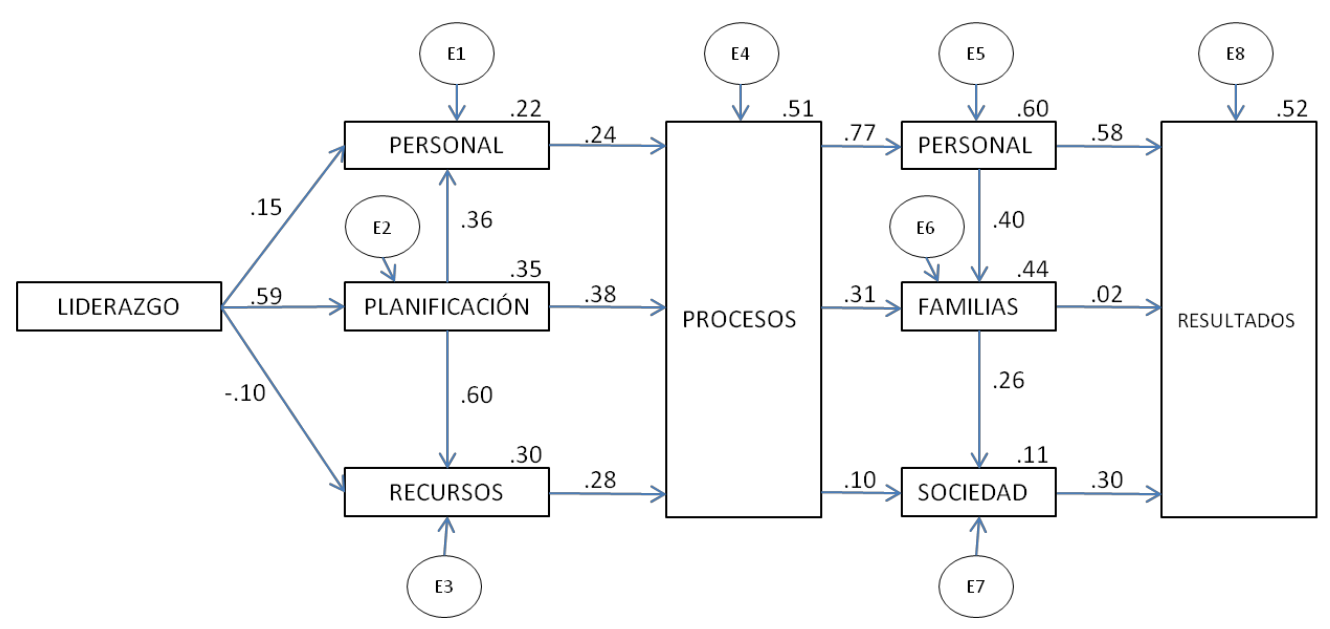

Figura 2. Modelo de ecuación estructural sobre la gestión de la convivencia escolar basado en el modelo EFQM

\section{Discusión}

Tras el análisis del modelo estructural, los resultados obtenidos apoyan la validez del modelo EFQM como marco de referencia para un análisis integral de la gestión de la convivencia escolar. Si bien, tal y como se ha indicado anteriormente, se sugiere la realización de réplicas de este mismo análisis incluyendo la totalidad de los factores, a través de la introducción de factores inclusivos en la ecuación.

En consecuencia, la validez de este modelo, si bien no puede confirmarse de forma definitiva, ni excluye a otros modelos de análisis globales alternativos o complementarios, corrobora los resultados de estudios como el de Calvo, Leal y Roldán (2006) en el que se revela el carácter sistémico del modelo de gestión y la necesidad de considerar a los agentes en su conjunto, tal y como planteaba Olweus (1993) al interpretar la convivencia escolar como un problema sistémico, y en consecuencia abordable desde todos los niveles de la comunidad escolar.

Se corrobora los resultados de otros estudios empíricos como los realizados por Eskildsen y Dahlgaard (2000) o Flynn et al. (1994), en los que se pone de manifiesto la influencia del factor Liderazgo, conducción y gobierno del Plan de Convivencia, actuando como fuerza motriz en la gestión de la convivencia escolar, sobre la planificación y gestión del personal.

Concretamente, las actuaciones en las que se materializa la conducción y gobierno del plan de convivencia responden a un modelo de acción democrático y participativo. A saber: recoger y discutir información de todos y con todos los sectores de la comunidad educativa, la existencia de procedimientos para explicar y difundir el plan de convivencia entre el alumnado, y entre el personal del centro, acotar tiempos y personas responsables para el análisis de la convivencia, identificar recursos humanos y materiales necesarios, la existencia de procedimientos para explicar y difundir el plan de convivencia entre las familias y entorno, elaborar el método y los instrumentos para la recogida de información, generar los cambios necesarios en actitudes y comportamientos y fomentar el funcionamiento de las estructuras de participación.

Por otro lado el factor Planificación, análisis de la realidad del centro, actúa como referencia para la gestión del personal, gestión de recursos y gestión de procesos. Este dato se acerca a los resultados aportados en el estudio de Campo, Fernández y Grisaleña (2005), que observan como un aspecto para la 
mejora de la convivencia, es documentar la realidad, a partir de un análisis compartido, entre la escuela y la comunidad escolar, de la convivencia concretando sus puntos fuertes y débiles.

Respecto a la gestión del personal se destaca como aspectos con influencia sobre los procesos todas aquellas cuestiones relativas a la formación del personal. Concretamente: formación del personal en mediación pacífica de conflictos, formación del personal en habilidades sociales e inteligencia emocional, formación del personal en organización y programación de la convivencia y formación del personal en aprendizaje dialógico.

Respecto a los Recursos, estos se centran fundamentalmente en los instrumentos y recursos que la administración facilita a los centros, para la mejora de la convivencia, así como aquellos elementos necesarios para la gestión de estos. Las variables son las siguientes: implicación del personal en las acciones formativas para el personal del centro en mejora de la convivencia escolar; Impulsar la formación de familiares en mejora de la convivencia escolar; participar en la celebración de actividades formativas conjuntas; organizar, clasificar y tener acceso al material para el profesor; impulsar el intercambio de experiencias entre centros; elaborar materiales propios en relación con la convivencia; elaborar y facilita recursos para la formación en mejora de la convivencia escolar; menor número de alumnos/as por aula. En este sentido Díaz-Aguado (2002) en un estudio realizado sobre las condiciones que mejoran la convivencia escolar, habla de poner a disposición del profesorado los medios que permitan desarrollar una convivencia democrática. Esta misma autora continúa diciendo que todas las innovaciones necesarias para educar la ciudadanía democrática exigen contar con condiciones que permitan a los profesores llevarlas a cabo. Conviene tener en cuenta en este sentido la necesidad de apoyar a los profesores, facilitando que adquieran las habilidades necesarias para conseguirlo y creando condiciones que posibiliten la cooperación entre profesores; sin caer en la frecuente tendencia a sobrevalorar las posibilidades de los profesores para desarrollar objetivos muy complejos sin los medios necesarios, ni en la tendencia contraria, infravalorando la capacidad de unos profesionales para adquirir las habilidades necesarias que permitan adaptar la educación a las exigencias de la situación actual.

El modelo de la ecuación confirma el papel mediador de los «procesos» entre los factores agentes y los resultados (Kanji y Tambi, 1999). Entre estos procesos se encuentran aspectos reconocidos por diversos estudios y postulados por diversos autores de referencia: la elaboración democrática de las normas (Caballero, 2010; Acosta, 2003; Cidad, 1996); las actividades dirigidas a favorecer la participación de las familias y el centro educativo (Caballero, 2010; Cascón, 2000; Esperanza, 2006); las actividades que se realizan en el aula de convivencia, favorecer la participación activa de los estudiantes (Esperanza, 2006); la atención a la diversidad y el aprendizaje significativo, la actuación de los compromisos de convivencia y los protocolos a seguir en la mediación pacifica de los conflictos (Esperanza, 2006; Díaz- Aguado, 2006; Torrego et al, 2000; Uranga, 1998; Torrego, 2003 y Otero, 2001).

Respecto a los resultados que obtiene el centro, es decir los logros que se están obteniendo respecto a las conductas contrarias y graves perjudiciales para la convivencia, parece que están influidos, especialmente, por la satisfacción que tenga el personal del centro con el plan de convivencia, y por los efectos que tenga en la sociedad.

Aunque los resultados de este estudio confirman la validez del modelo EFQM para un análisis integral de la convivencia escolar, diferenciando entre factores agentes y resultados, resulta preciso insistir en la necesidad de incorporar en la ecuación estructural factores más inclusivos y en consecuencia aumentar el número de factores. Como resultado de todo ello se fortalecería la validación del modelo como instrumento para el análisis de la convivencia escolar.

Asimismo, para avanzar en el proceso de validación resulta necesaria la aplicación del cuestionario en otras muestras y contextos diversos que permitan contrastar el peso de cada factor sobre la varianza de otros factores. Tampoco puede olvidarse el proceso de validez teórica de los factores analizados. Téngase en consideración que el contenido y materialización de los factores a estudio responden a una interpretación del fenómeno con base epistemológica, amparada en la experiencia de profesionales y avalada por resultados de investigaciones más básicas. En consecuencia, ante esta "movilidad" teórica, el estudio básico de los factores implicados no debe perderse como referencia, como insumos que permitan una construcción teórica articulada. 
Finalmente, como también se ha comentado anteriormente, la opción por el modelo EFQM para el estudio integral de la convivencia escolar no excluye a otros modelos de análisis, más bien se pretende estimular a la comunidad científica a la aplicación de otros modelos alternativos e integrales que permitan el desarrollo de un discurso más global del fenómeno de studio.

\section{Referencias}

Acosta, A. (2003). Resolución de conflictos y regulación de sentimientos. En Molina, B., Muñoz, F. y Jiménez, F. Actas del I Congreso Hispanoamericano de Educación y Cultura de Paz, Granada., 293-304

Bardisa, T. (2001). La participación en las organizaciones escolares. En García de Cortázar y cols. Consensos y conflictos en los centros docentes no universitarios. Madrid: UNED. Colección Varia.

Biehl, R.E. (2000). Customer-supplier analysis in educational change. Quality Management Journal, 2, 22-39.

Caballero, M.J. (2010). Convivencia Escolar. Un estudio sobre buenas prácticas. Paz y Conflictos, 3, 154-169.

Calvo, A., Leal, A. y Roldán, J. (2006). Using enablers of the EFQM model to manage institutions of higher education. Quality Assurance in Education, 14, 99-122.

Campo, A., Fernández, A y Grisaleña, J. (2005). La Convivencia en los Centros de Secundaria: Un estudio de casos. Revista Iberoamericana de Educación, 38, 121-145

Cascón, P. (2000). Educar en y para el conflicto. Cuadernos de Pedagogía, 287, 61-66.

Cidad, E. (1996). Modificación de conducta en el aula e integración escolar. Madrid: U.N.E.D.

Cuevas, F.J. (2006). Trabajando desde las redes comunitarias para la resolución de conflictos en la escuela. Comunicación presentada en las II jornadas internacionales sobre políticas educativas para la sociedad del conocimiento. Granada.

Decreto 19/2007, de 23 de enero, por el que se adoptan medidas para la promoción de la Cultura de Paz y la Mejora de la Convivencia en los Centros Educativos sostenidos con fondos públicos. (BOJA, No 25, del 2 febrero 2007).

Detert, J.R., Jenni, R. (2000). An instrument for measuring quality practice in education. Quality Management Journal, 7, 20-37.

Díaz-Aguado, M.J. (2002). Por una Cultura de la convivencia democrática. Revista Interuniversitaria de Formación del Profesorado. 44, 55-78.

Díaz- Aguado, M.J. (2006). Mejorar la convivencia en el aula a través del aprendizaje cooperativo y el currículum de la no-violencia. Recuperado de http://mariajosediaz-aguado.tk.

Estudio Estatal sobre convivencia escolar en la Educación Secundaria Obligatoria (2010). Observatorio Convivencia Escolar.

Eskildsen, J.K. y Dahlgaard, J.J. (2000). A causal model for employee satisfaction. Total Quality Management, 11, 81-94.

Esperanza, J. (2006). La respuesta global: programas, instrumentos y actitudes. Cuadernos de pedagogía, $359,66-72$.

European Foundation for Quality Management. (2003). Modelo EFQM de Excelencia. Brussels: European Foundation for Quality Management. 
Fernández-Baena, F.J., Trianes, M.V., De la Morena, M.L., Escobar, M., Infante, L. y Blanca, M. J. (2011). Propiedades psicométricas de un cuestionario para la evaluación de la violencia cotidiana entre iguales en el contexto escolar. Anales de Psicología, 27(1), 102-108.

Fernández-García, I. (2008). Los programas de ayuda para la mejora de la convivencia en instituciones educativas. Bordón, 60(4), 137-150.

Flynn, B., Schroeder, R.G. y Sakakibara, S. (1994). A framework for quality management research and an associated measurement instrument. Journal of Operations Management, 4, 339-66.

Kanji, G. K., Tambi, A.M y Wallace, W. (1999). A comparative study of quality practices in higher education institutions in US and Malaysia. Total Quality Management,3, 357-71.

García-Raga, L. y López-Martín, R. (2011). Convivir en la escuela. Una propuesta para su aprendizaje por competencias. Revista de Educación, 356, 531-555.

Mateo, J. (2000). La evaluación educativa sus prácticas y otras metáforas. ICE de la Universitat de Barcelona-Horsori. Barcelona.

Mel Farrar (2000). Structuring success: a case study in the use of the EFQM Excellence Model in School improvement. Total Quality Management, 4-6, 691-696.

Montano, C.B. y Glenn, H.U. (1999). Total Quality Management in higher education. Quality progress, August, 52-9.

Olweus, D. (1993). Bullying at school: What Know and what we can do. Oxford: Blackwell.

Orden de 20 de junio de 2011, por la que se adoptan medidas para la promoción de la convivencia en los centros docentes sostenidos con fondos públicos y se regula el derecho de las familias a participar en el proceso educativo de sus hijos e hijas. (BOJA, No 132, del 7 de julio de 2011).

Ortega, R., Monje, M. y Córdoba, F. (2009). La actualidad de los modelos legislativos de convivencia: un metanálisis. Educar, 43, 81-91.

Ortega, R. y Del Rey, R. (2006). La violencia escolar: estrategias de prevención. Barcelona: Graó.

Osseo-Asare, A.E. y Longbottom, D. (2002). The need for education and training in the use of the EFQM model for quality management in UK higher education institutions. Quality Assurance in Education, 1, 25-35.

Otero, V. (2001). Convivencia escolar: Problemas y soluciones. Revista Complutense de educación, 1, 295-318.

Pedrero, E. (2011). La situación de la convivencia escolar en España: análisis del estudio estatal sobre convivencia escolar en la Educación Secundaria Obligatoria. En J. J. Leiva y R. Borrero (Eds.). Interculturalidad y Escuela (pp. 13-39). Barcelona: Octaedro.

Saraiva, P.M., Rosa, M.J. y d'Oreyi, J.L. (2003). Applying an excellence model to schools. Quality progress, $11,46$.

Spanbauer, S.J. (1995). Reactivating higher education with total quality management: using quality and productivity concepts, techniques and tools to improve higher education. Total Quality Management,5/6, 519-38.

Taffinder, P. (1995). The New Leaders: Achieving Corporate Transformation through Dynamic Leadership. London: Kogan Page.

Torrego, J.C.(Coord.) (2003). Mediación de conflictos en instituciones educativas. Manual para la formación de mediadores. Madrid: Narcea. 
Análisis integral de la gestión de la convivencia escolar. Una propuesta de evaluación

Tuvilla, J. (2004). Convivencia escolar y resolución pacifica de conflictos. Sevilla: Consejería de Educación y Ciencia.

Uranga, M. (1998). Mediación, negociación y habilidades para el conflicto en el marco escolar. Barcelona: Graó.

Weller, L.D. (2000). School attendance problems: using the TQM tools to identify root causes. Journal of Educational Administration, 1, pp. 64-72.

Winn, B.A y Cameron, K.S. (1998). Organizational quality: an examination of the Baldrige National Quality Framework. Research in Higher Education, 39 , pp. 491-512.

Zairi, M. (1994). Leadership in TQM implementation: some case examples. The TQM Magazine, 6, pp. 9-16

Fecha de recepción / Received: 08/06/2015

Fecha de aceptación / Accepted: 30/06/2015 


\section{ANEXO}

1. LIDERAZGO
Valore aquí la labor del Equipo Directivo y la Comisión de Convivencia respecto a la gestión de la
convivencia escolar
1.1 El Equipo Directivo demuestra visiblemente su compromiso con la Cultura de Paz y la mejora de la
convivencia

O Nada, 1 Algo, 2 Bastante, 3 Mucho, ¿? No sabe, no contesta (escriba una X donde proceda)

1. Conoce los aspectos claves del plan de convivencia

2. Define un modelo de convivencia

3. Genera los cambios necesarios en actitudes y comportamientos

4. Facilita actividades para la mejora continua.

5. Su capacidad de liderazgo es reconocida por el personal del centro.

\begin{tabular}{|l|l|l|l|l|}
\hline 0 & 1 & 2 & 3 & $¿ ?$ \\
\hline 0 & 1 & 2 & 3 & $¿ ?$ \\
\hline 0 & 1 & 2 & 3 & $¿ ?$ \\
\hline 0 & 1 & 2 & 3 & $¿ ?$ \\
\hline 0 & 1 & 2 & 3 & $¿ ?$ \\
\hline
\end{tabular}

1.2. El Equipo Directivo, en colaboración con el orientador, coordina la elaboración del plan de convivencia.

0 Nada, 1 Algo, 2 Bastante, 3 Mucho, ¿? No sabe, no contesta (escriba una X donde proceda)

6. Define cómo se elaborará el plan de convivencia en el centro educativo.

7. Establece estrategias e instrumentos para facilitar la participación en la elaboración del plan.

8. Fomenta el funcionamiento de las estructura de participación (asamblea de aula, junta de delegados/as, etc.)

\begin{tabular}{|l|l|l|l|l|}
\hline 0 & 1 & 2 & 3 & $¿ ?$ \\
\hline 0 & 1 & 2 & 3 & $\dot{?} ?$ \\
\hline 0 & 1 & 2 & 3 & $\dot{?} ?$ \\
\hline
\end{tabular}

1.3. La Comisión de Convivencia canaliza las iniciativas de todos los sectores de la comunidad educativa para mejorar la convivencia, el respeto mutuo, así como promover la cultura de paz y la resolución pacífica de los conflictos.

0 Nada, 1 Algo, 2 Bastante, 3 Mucho, ¿? No sabe, no contesta (escriba una $X$ donde proceda)

9. Elabora el método y los instrumentos para la recogida de información.

10. Recoge y discute información de todos y con todos los sectores de la comunidad educativa.

11. Acota los tiempos y personas responsables para el análisis de la convivencia.

12. Identifica los recursos humanos y materiales necesarios.

\begin{tabular}{|l|l|l|l|l|}
\hline 0 & 1 & 2 & 3 & $¿ ?$ \\
\hline 0 & 1 & 2 & 3 & $¿ ?$ \\
\hline 0 & 1 & 2 & 3 & $¿ ?$ \\
\hline 0 & 1 & 2 & 3 & $¿ ?$ \\
\hline
\end{tabular}

1.4. La Comisión de Convivencia impulsa las medidas preventivas necesarias para garantizar los derechos de todos los miembros de la comunidad educativa y el cumplimiento de las normas de convivencia del centro

O Nada, 1 Algo, 2 Bastante, 3 Mucho, ¿? No sabe, no contesta (escriba una X donde proceda) Existen procedimientos para explicar y difundir el plan de convivencia (revistas informativas, sesiones de tutorías, reuniones, etc.) entre:
13. El personal del centro
14. El alumnado
15. Familias y entorno

\begin{tabular}{|l|l|l|l|l|}
\hline 0 & 1 & 2 & 3 & $¿ ?$ \\
\hline 0 & 1 & 2 & 3 & $¿ ?$ \\
\hline 0 & 1 & 2 & 3 & $¿ ?$ \\
\hline
\end{tabular}

2. PLANIFICACIÓN Y ESTRATEGIA

Valore aquí como programáis la convivencia escolar

2.1. Identificación de los factores relacionados con la ubicación del centro que condicionan el estado de la convivencia escolar (ejemplo: accesos, rivalidad entre grupos, barrios, zonas deprimidas socialmente...)

O Nada, 1 Algo, 2 Bastante, 3 Mucho, ¿? No sabe, no contesta (escriba una X donde proceda) 16. Se identifican las características del centro: espacios del centro, distribución, uso, repercusión en la convivencia

17. Se identifican las características del Profesorado, PAS: estabilidad, nivel de desarrollo profesional.

\begin{tabular}{|l|l|l|l|l|}
\hline 0 & 1 & 2 & 3 & $i ?$ \\
\hline 0 & 1 & 2 & 3 & $i ?$ \\
\hline
\end{tabular}


18. Se identifican las características de las familias: nivel sociocultural, económico, laboral, grado de colaboración con el centro.

19. Se identifican las características del alumnado: inmigrante, absentista, con dificultades de aprendizaje, índice de fracaso escolar.

\subsection{Análisis del clima educativo del centro.}

O Nada, 1 Algo, 2 Bastante, 3 Mucho, ¿’? No sabe, no contesta (escriba una $X$ donde proceda)

20. Procesos de enseñanza-aprendizaje basados en la cooperación.

21. Se impulsa el diálogo, la ayuda mutua, la negociación e interdependencia positiva en el aula y en el centro.

22. Se impulsa las relaciones positivas, la confianza y respeto entre los miembros de la comunidad educativa.

23. Existe una verdadera cooperación entre todos los miembros de la comunidad educativa

24. Todos los miembros de la comunidad educativa se implican en la planificación y gestión de la convivencia.

\subsection{Análisis de las prácticas educativas que tienen relación directa con la convivencia}

O Nada, 1 Algo, 2 Bastante, 3 Mucho, ¿̇? No sabe, no contesta (escriba una X donde proceda)

$$
\text { El profesorado sabe reaccionar ante conductas indeseadas y disruptivas. } \quad \begin{array}{rl|l|l|l|l|}
\hline \mathbf{0} & \mathbf{1} & \mathbf{2} & \mathbf{3} & \dot{\mathrm{c}} \text { ? }
\end{array}
$$

\subsection{Actuaciones realizadas por el centro para la gestión de la} convivencia

O Nada, 1 Algo, 2 Bastante, 3 Mucho, ¿̇? No sabe, no contesta (escriba una X donde proceda)

26. El Centro incorpora metodologías pedagógicas que favorezcan la convivencia: ayuda entre iguales, tutorías compartida, aprendizaje cooperativo, etc.

27. Se forma en inteligencia emocional y social en el contexto del desarrollo del currículo

28. Se han realizado programas de formación del profesorado en convivencia.

29. Se ha realizado programas de dinamización sociocomunitaria

30. Se han realizado campañas de información y sensibilización del Plan de convivencia.

31. Todos los programas se revisan, actualizan, y se proponen mejoras cuando finalizan

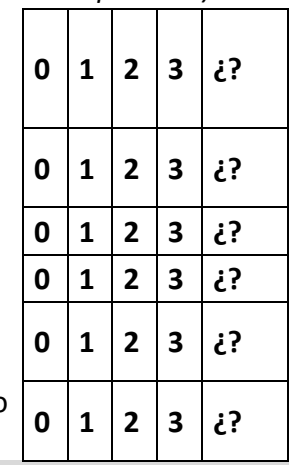

2.5. Procedimiento de elaboración del plan de convivencia.

O Nada, 1 Algo, 2 Bastante, 3 Mucho, ¿̇? No sabe, no contesta (escriba una X donde proceda)

32. La Comisión realiza el diagnostico de la situación de la convivencia en el centro.

33. La Comisión de convivencia, elabora una propuesta de contenidos del

plan.

34. El Equipo Directivo da a conocer el plan a la comunidad educativa e impulsa sus aportaciones.

35. El equipo directivo elabora una propuesta del plan de convivencia del centro.

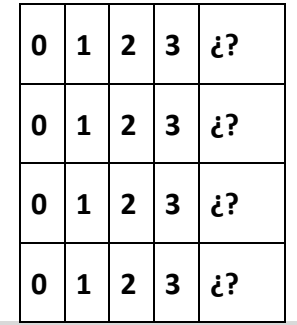

\subsection{Planificación de la mediación de conflictos.}

o Nada, 1 Algo, 2 Bastante, 3 Mucho, ¿'? No sabe, no contesta (escriba una $X$ donde proceda)

36. Existe un plan de mediación de conflictos.

\begin{tabular}{|l|l|l|l|l|}
\hline $\mathbf{0}$ & $\mathbf{1}$ & $\mathbf{2}$ & $\mathbf{3}$ & $\dot{\imath} ?$ \\
\hline
\end{tabular}

El plan de mediación de conflictos recoge las siguientes líneas de actuación:

37. Formación de la comunidad educativa en competencias relacionadas con la resolución pacífica de conflictos.

38. Existe protocolos para la mediación pacífica de conflictos.

39. Creación de agentes de mediación.

\begin{tabular}{|l|l|l|l|l|}
\hline 0 & 1 & 2 & 3 & $\dot{ } ?$ \\
\hline 0 & 1 & 2 & 3 & $\dot{ } ?$ \\
\hline 0 & 1 & 2 & 3 & $\dot{ } ?$ \\
\hline
\end{tabular}


2.7. El plan de convivencia recoge los contenidos de los compromisos de convivencia de las familias, alumnos y del centro.

O Nada, 1 Algo, 2 Bastante, 3 Mucho, ¿'? No sabe, no contesta (escriba una $X$ donde proceda)

40. Se determina el perfil del alumnado al que va dirigido.

41. Se concreta un protocolo de actuación.

42. Se establecen los mecanismos de seguimiento y evaluación.

\begin{tabular}{|l|l|l|l|l|}
\hline 0 & 1 & 2 & 3 & $\dot{\imath} ?$ \\
\hline 0 & 1 & 2 & 3 & $\dot{\imath} ?$ \\
\hline 0 & 1 & 2 & 3 & $\dot{\imath} ?$ \\
\hline
\end{tabular}

2.8. Planificación del aula de convivencia.

o Nada, 1 Algo, 2 Bastante, 3 Mucho, ¿̇? No sabe, no contesta (escriba una X donde proceda)

43. Se definen los principios de actuación pedagógica del aula de convivencia

(Finalidades, objetivos).

44. Se establecen las condiciones de acceso.

45. Se establece el procedimiento de derivación.

46. Se planifica la organización del aula y sus recursos.

\begin{tabular}{|l|l|l|l|l|}
\hline 0 & 1 & 2 & 3 & $\dot{c} ?$ \\
\hline 0 & 1 & 2 & 3 & $\dot{ } ?$ \\
\hline 0 & 1 & 2 & 3 & $\dot{c} ?$ \\
\hline 0 & 1 & 2 & 3 & $\dot{ } ?$ \\
\hline
\end{tabular}

3. GESTIÓN DE PERSONAL

Valore aquí cómo el Centro Educativo aprovecha al máximo las aptitudes de su personal para la mejora de la convivencia escolar.

3.1. Plan de trabajo de la comisión de convivencia.

O Nada, 1 Algo, 2 Bastante, 3 Mucho, ¿'? No sabe, no contesta (escriba una $X$ donde proceda)

47. Se explicita para cada una de las actividades: las personas responsables, los recursos, la metodología, contenido, espacio y temporalización

48. Se incorporan procedimientos y momentos para la revisión y evaluación del plan.

\begin{tabular}{|l|l|l|l|l|}
\hline 0 & 1 & 2 & 3 & $\dot{ } ?$ \\
\hline 0 & 1 & 2 & 3 & $i ?$ \\
\hline
\end{tabular}

3.2. Definición de funciones y tareas del Educador Social. (si es el caso)

O Nada, 1 Algo, 2 Bastante, 3 Mucho, ¿'? No sabe, no contesta (escriba una X donde proceda)

49. El Educador/a Social desarrolla las tareas de relación entre el centro educativo y las familias del alumnado.

50. El Educador/a Social realiza las funciones de intermediación educativa entre el alumnado y el resto de la comunidad educativa.

51. El Educador/a Social colabora en el desarrollo de programas para la educación en valores y en la mejora de la convivencia escolar.

\begin{tabular}{|c|c|c|c|c|}
\hline 0 & 1 & 2 & 3 & ¿'? \\
\hline 0 & 1 & 2 & 3 & ¿'? \\
\hline 0 & 1 & 2 & 3 & ¿'? \\
\hline
\end{tabular}

3.3. Formación de la comunidad educativa para la mejora de la convivencia.

o Nada, 1 Algo, 2 Bastante, 3 Mucho, ¿'? No sabe, no contesta (escriba una X donde proceda)

Se forma al personal en los siguientes aspectos:

52. Organización y programación de la convivencia.

53. Mediación pacífica de conflictos.

54. Habilidades sociales e inteligencia emocional.

55. Aprendizaje dialógico.

56. Educación para la Paz.

\begin{tabular}{|l|l|l|l|l|}
\hline 0 & 1 & 2 & 3 & $\dot{ } ?$ \\
\hline 0 & 1 & 2 & 3 & $\dot{\imath} ?$ \\
\hline 0 & 1 & 2 & 3 & $\dot{¿} ?$ \\
\hline 0 & 1 & 2 & 3 & $\dot{c} ?$ \\
\hline 0 & 1 & 2 & 3 & $\dot{ } ?$ \\
\hline
\end{tabular}

4. RECURSOS

Valore aquí cómo el centro educativo aprovecha los recursos y apoyos para la mejora de la convivencia escolar.

4.1. Instrumentos y recursos que la administración facilita a los centros para la mejora de la convivencia.

O Nada, 1 Algo, 2 Bastante, 3 Mucho, ¿'? No sabe, no contesta (escriba una $X$ donde proceda)

57. Se cuenta con profesorado de apoyo específico.

58. Existe menor número de alumnos/as por aula.

59. Se cuenta con el personal funcionario con la titulación de Educador/a Social.

60. Se elabora y facilita recursos para la formación en mejora de la convivencia escolar.

\begin{tabular}{|l|l|l|l|l|}
\hline 0 & 1 & 2 & 3 & $\dot{\imath} ?$ \\
\hline 0 & 1 & 2 & 3 & $\dot{\imath} ?$ \\
\hline 0 & 1 & 2 & 3 & $\dot{¿} ?$ \\
\hline 0 & 1 & 2 & 3 & $\dot{\imath} ?$ \\
\hline
\end{tabular}


61. El personal se implica en las acciones formativas para el personal del centro en mejora de la convivencia escolar.

62. Se Impulsa la formación de familiares en mejora de la convivencia escolar.

63. Se participa en la celebración de actividades formativas conjuntas.

64. Se impulsa el intercambio de experiencias entre centros.

\begin{tabular}{|l|l|l|l|l|}
\hline 0 & 1 & 2 & 3 & $\dot{?} ?$ \\
\hline 0 & 1 & 2 & 3 & $\dot{\imath} ?$ \\
\hline 0 & 1 & 2 & 3 & $\dot{?} ?$ \\
\hline 0 & 1 & 2 & 3 & $\dot{?} ?$ \\
\hline
\end{tabular}

\subsection{Organización y gestión de recursos.}

O Nada, 1 Algo, 2 Bastante, 3 Mucho, ¿̇? No sabe, no contesta (escriba una X donde proceda)

65. Se elaboran materiales propios en relación con la convivencia.

66. Existen espacios alternativos al aula ordinaria para casos disruptivos (por ejemplo aula de convivencia).

67. El material está organizado, clasificado y accesible para el profesor.

\begin{tabular}{|l|l|l|l|l|}
\hline 0 & 1 & 2 & 3 & $¿ ?$ \\
\hline 0 & 1 & 2 & 3 & $¿ ?$ \\
\hline 0 & 1 & 2 & 3 & $¿ ?$ \\
\hline
\end{tabular}

\subsection{Funcionamiento y personas responsables del aula de convivencia}

0 Nada, 1 Algo, 2 Bastante, 3 Mucho, ¿̇? No sabe, no contesta (escriba una $X$ donde proceda)

68. Se definen "requisitos" del profesorado que atienda el aula de convivencia.

69. El centro asigna al resto de profesorado tareas de atención y control del aula de convivencia.

70. Se establece el horario de funcionamiento del aula de convivencia.

71. Se cuenta con la colaboración del Educador/a Social.

72. El departamento de orientación o EOE programa las acciones didácticas.

73. Las personas responsables del aula realizan los seguimientos de asistencia del alumnado derivado a la misma.

\begin{tabular}{|l|l|l|l|l|}
\hline 0 & 1 & 2 & 3 & $\dot{\imath} ?$ \\
\hline 0 & 1 & 2 & 3 & $\dot{\imath} ?$ \\
\hline 0 & 1 & 2 & 3 & $\dot{\imath} ?$ \\
\hline 0 & 1 & 2 & 3 & $\check{?} ?$ \\
\hline 0 & 1 & 2 & 3 & $\dot{\imath} ?$ \\
\hline 0 & 1 & 2 & 3 & $¿ ?$ \\
\hline
\end{tabular}

\subsection{Instalaciones y material didáctico del aula de convivencia.}

O Nada, 1 Algo, 2 Bastante, 3 Mucho, ¿’? No sabe, no contesta (escriba una $X$ donde proceda)

74. El aula de convivencia tiene un tamaño adecuado.

75. El mobiliario está dispuesto de forma que facilita el diálogo y la reflexión.

76. Su ubicación la hace accesible y próxima.

77. Existen materiales suficiente y de calidad para su uso didáctico.

\begin{tabular}{|l|l|l|l|l|}
\hline 0 & 1 & 2 & 3 & $\dot{\imath} ?$ \\
\hline 0 & 1 & 2 & 3 & $\dot{\imath} ?$ \\
\hline 0 & 1 & 2 & 3 & $\dot{¿} ?$ \\
\hline 0 & 1 & 2 & 3 & $\dot{\imath} ?$ \\
\hline
\end{tabular}

\section{PROCESOS}

Valore aquí la forma de proceder y actuar que permiten lograr los resultados del plan de convivencia.

\subsection{Elaboración democrática de las normas.}

o Nada, 1 Algo, 2 Bastante, 3 Mucho, ¿'? No sabe, no contesta (escriba una X donde proceda)

78. Las normas son elaboradas contando con la participación de toda la comunidad educativa.

79. Se hace participar a toda la comunidad educativa en la identificación de los problemas de convivencia.

80. Se indaga sobre las causas de los problemas para establecer las normas.

81. Existen eficaces mecanismos para la difusión de las normas.

82. Las normas se revisan periódicamente.

\begin{tabular}{|l|l|l|l|l|}
\hline 0 & 1 & 2 & 3 & $\dot{\imath} ?$ \\
\hline 0 & 1 & 2 & 3 & $\dot{\imath} ?$ \\
\hline 0 & 1 & 2 & 3 & $\dot{?} ?$ \\
\hline 0 & 1 & 2 & 3 & $\dot{\imath} ?$ \\
\hline 0 & 1 & 2 & 3 & $\dot{?} ?$ \\
\hline
\end{tabular}

\subsection{Actividades encaminadas a facilitar la integración y la participación del alumnado.}

O Nada, 1 Algo, 2 Bastante, 3 Mucho, ¿̇? No sabe, no contesta (escriba una $X$ donde proceda)

83. Se hacen actividades de acogida en la que se informe sobre las normas para una convivencia pacífica.

84. Se especifica los derechos y deberes del alumnado y las correcciones y medidas disciplinarias que en su caso se aplicarían.

85. Se ofrece orientación individual.

86. Se hacen talleres de resolución de conflictos para el alumnado.

87. Se trabaja el desarrollo de habilidades emocionales y de competencia social.

88. Se hacen programas de refuerzo educativo.

\begin{tabular}{|l|l|l|l|l|}
\hline 0 & 1 & 2 & 3 & $\dot{?}$ \\
\hline 0 & 1 & 2 & 3 & $\dot{\imath} ?$ \\
\hline 0 & 1 & 2 & 3 & $\dot{?} ?$ \\
\hline 0 & 1 & 2 & 3 & $\dot{?} ?$ \\
\hline 0 & 1 & 2 & 3 & $\dot{?} ?$ \\
\hline 0 & 1 & 2 & 3 & $\dot{?} ?$ \\
\hline
\end{tabular}


5.3. Actividades dirigidas a favorecer la participación de las familias y el centro educativo.

O Nada, 1 Algo, 2 Bastante, 3 Mucho, ¿'? No sabe, no contesta (escriba una X donde proceda)

89. Se establecen mecanismos para garantizar el conocimiento de la familia de las normas de convivencia.

90. Se estimula la participación de las familias en la vida del centro.

91. Se realizan acciones formativas para las familias (Resolución pacífica de conflictos, programas de mediación, etc.)

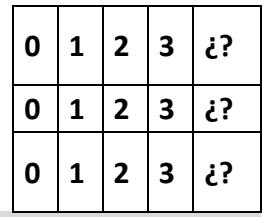

\subsection{Se favorece en el aula:}

O Nada, 1 Algo, 2 Bastante, 3 Mucho, ¿'? No sabe, no contesta (escriba una X donde proceda)

92. El aprendizaje cooperativo.

93. La participación activa de los estudiantes.

94. La atención a la diversidad.

95. El aprendizaje significativo.

\begin{tabular}{|l|l|l|l|l|}
\hline 0 & 1 & 2 & 3 & $\dot{\imath} ?$ \\
\hline 0 & 1 & 2 & 3 & $\dot{\imath} ?$ \\
\hline 0 & 1 & 2 & 3 & $\dot{ } ?$ \\
\hline 0 & 1 & 2 & 3 & $\dot{ } ?$ \\
\hline
\end{tabular}

5.6. Actividades en el aula de convivencia.

O Nada, 1 Algo, 2 Bastante, 3 Mucho, ¿ं? No sabe, no contesta (escriba una $X$ donde proceda)

96. Se realizan actividades que faciliten la reflexión del alumno/a sobre su comportamiento problemático.

97. Se hacen actividades que mejoren la actitud del alumno/a y garantice su proceso educativo.

98. Las actividades son adaptadas en función de las características y necesidades de cada alumno

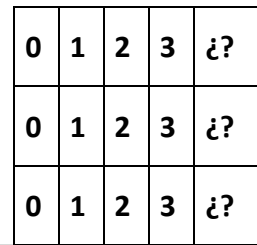

5.7. Actuación de los compromisos de convivencia.

O Nada, 1 Algo, 2 Bastante, 3 Mucho, ¿'? No sabe, no contesta (escriba una $X$ donde proceda)

99. El tutor analiza junto a la familia el progreso y cumplimiento de los compromisos.

100. Se acuerdan encuentros periódicos entre tutor y familiares para el seguimiento.

101. Se hace partícipe a la Comisión del seguimiento de los compromisos de convivencia.

5.8. Mediación para la resolución pacífica de los conflictos.

O Nada, 1 Algo, 2 Bastante, 3 Mucho, ¿ं? No sabe, no contesta (escriba una X donde proceda)

102. Se identifica y define el conflicto.

103. Se clarifica los intereses y sentimientos de las partes en el conflicto.

104. Se generan alternativas para dar posibles soluciones (lluvias de ideas).

105. Se evalúa las alternativas y se toman decisiones. (por mutuo acuerdo, se eliminan las que parezcan inviable, se integran las que puedan utilizarse)

106. Se crean formas de cumplimiento de la solución (qué hay que hacer y quién lo va a hacer)

107. Queda claro el compromiso de cada persona (cómo y cuándo)

108. Se establecen acuerdos claros.

109. Cada parte expresa lo que aprendió y cómo puede aplicarlo a otros problemas.

110. Se expresa cómo se han sentido y cómo se sienten con las soluciones acordadas.

111. Se reconoce el esfuerzo y la contribución de las partes en la resolución del conflicto.

112. Se hace un seguimiento para evaluar el cumplimiento de los acuerdos.

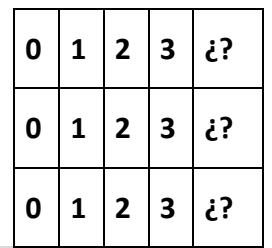

\begin{tabular}{|c|c|c|c|c|}
\hline 0 & 1 & 2 & 3 & ¿'? \\
\hline 0 & 1 & 2 & 3 & ¿'? \\
\hline 0 & 1 & 2 & 3 & ¿? \\
\hline 0 & 1 & 2 & 3 & ¿? \\
\hline 0 & 1 & 2 & 3 & ¿'? \\
\hline 0 & 1 & 2 & 3 & ¿? \\
\hline 0 & 1 & 2 & 3 & ¿? \\
\hline 0 & 1 & 2 & 3 & ¿'? \\
\hline 0 & 1 & 2 & 3 & ¿'? \\
\hline 0 & 1 & 2 & 3 & ¿'? \\
\hline 0 & 1 & 2 & 3 & ¿'? \\
\hline
\end{tabular}


1. Conocimiento que tienen las familias del plan de convivencia.

2. Identificación de las familias con el plan de convivencia.

\begin{tabular}{|c|c|c|c|c|}
\hline 0 & 1 & 2 & 3 & ¿? \\
\hline 0 & 1 & 2 & 3 & ¿'? \\
\hline
\end{tabular}

\subsection{Satisfacción que tienen las familias ( según usted lo percibe) respecto a:}

0 Nada, 1 Algo, 2 Bastante, 3 Mucho, ¿'? No sabe, no contesta (escriba una $X$ donde proceda)

3. El clima de convivencia y las relaciones humanas.

4. Resultados del plan.

5. Organización y funcionamiento general del plan.

6. Accesibilidad que tienen los familiares para participar en el plan de convivencia.

7. Los horarios de los distintos servicios del plan de convivencia.

8. La información de los distintos servicios del plan de convivencia.

\begin{tabular}{|l|l|l|l|l|}
\hline 0 & 1 & 2 & 3 & $\dot{ } ?$ \\
\hline 0 & 1 & 2 & 3 & $\dot{?} ?$ \\
\hline 0 & 1 & 2 & 3 & $\dot{ } ?$ \\
\hline 0 & 1 & 2 & 3 & $\dot{?} ?$ \\
\hline 0 & 1 & 2 & 3 & $\dot{ } ?$ \\
\hline 0 & 1 & 2 & 3 & $\dot{2} ?$ \\
\hline
\end{tabular}

6.3. Satisfacción las familias con las actuaciones del plan de convivencia. ( según usted lo percibe) Respecto a:

O Nada, 1 Algo, 2 Bastante, 3 Mucho, ¿̇? No sabe, no contesta (escriba una $X$ donde proceda)

9. Aula de convivencia.

10. Compromisos de convivencia.

11. Mediación en conflictos.

12. Actividades realizadas encaminadas a facilitar la integración y la participación del alumnado.

13. Actividades realizadas dirigidas a favorecer la relación de las familias y el centro educativo

14. Actividades realizadas dirigidas a prevenir la violencia en el ámbito comunitario.

15. Medidas adoptadas por el centro de carácter organizativo.

\begin{tabular}{|c|c|c|c|c|}
\hline U & 1 & 2 & 3 & ¿? \\
\hline 0 & 1 & 2 & 3 & ¿? \\
\hline 0 & 1 & 2 & 3 & ¿? \\
\hline 0 & 1 & 2 & 3 & ¿? \\
\hline 0 & 1 & 2 & 3 & ¿'? \\
\hline 0 & 1 & 2 & 3 & ¿? \\
\hline 0 & 1 & 2 & 3 & ¿? \\
\hline
\end{tabular}

6.4. Medidas complementarias relativas a la satisfacción de los familiares del plan de convivencia. (de acuerdo con datos objetivos)

O Nada, 1 Algo, 2 Bastante, 3 Mucho, ¿̇? No sabe, no contesta (escriba una $X$ donde proceda)

16. Valora los resultados del plan en las sucesivas evaluaciones.

17. Las entrevistas solicitadas por las familias han sido atendidas.

18. Las quejas presentadas por las familias han sido atendidas.

\begin{tabular}{|l|l|l|l|l|}
\hline 0 & 1 & 2 & 3 & $\dot{c} ?$ \\
\hline 0 & 1 & 2 & 3 & $\dot{c} ?$ \\
\hline 0 & 1 & 2 & 3 & $\dot{ } ?$ \\
\hline
\end{tabular}

\section{SATISFACCIÓN DEL PERSONAL.}

Valore aquí al plan de convivencia del centro educativo como personal que es usted del mismo.

7.1. Percepción que tiene el personal docente y de administración y servicios de su plan de convivencia.

O Nada, 1 Algo, 2 Bastante, 3 Mucho, ¿ं? No sabe, no contesta (escriba una $X$ donde proceda)

19. Conocimiento que tienen del plan de convivencia.

20. Identificación con el plan de convivencia.

21. Satisfacción, respecto al plan de convivencia.

22. Satisfacción con los resultados del plan de convivencia.

23. Motivación para implicarse en el plan de convivencia escolar.

24. Satisfacción que tiene el personal respecto a la organización y funcionamiento del plan.

25. Satisfacción del personal con la participación en la toma de decisiones.

26. Satisfacción del personal con el tipo de liderazgo que se ejerce en el plan.

27. Satisfacción del personal con los recursos con que dispone el centro para desarrollar las funciones referidas al plan de convivencia.

\begin{tabular}{|c|c|c|c|c|}
\hline 0 & 1 & 2 & 3 & ¿? \\
\hline 0 & 1 & 2 & 3 & $\dot{d} ?$ \\
\hline 0 & 1 & 2 & 3 & ¿'? \\
\hline 0 & 1 & 2 & 3 & ¿'? \\
\hline 0 & 1 & 2 & 3 & ¿'? \\
\hline 0 & 1 & 2 & 3 & $\dot{d} ?$ \\
\hline 0 & 1 & 2 & 3 & $\dot{2} ?$ \\
\hline 0 & 1 & 2 & 3 & ¿? \\
\hline 0 & 1 & 2 & 3 & ¿? \\
\hline
\end{tabular}




\subsection{Satisfacción del personal respecto a:}

0 Nada, 1 Algo, 2 Bastante, 3 Mucho, ¿ं? No sabe, no contesta (escriba una $X$ donde proceda)

28. Aula de convivencia.

29. Compromisos de convivencia.

30. Mediación en conflictos.

31. Actividades realizadas encaminadas a facilitar la integración y la participación del alumnado.

32. Actividades realizadas dirigidas a favorecer la relación de las familias y el centro educativo.

33. Actividades realizadas dirigidas a prevenir la violencia en el ámbito comunitario.

34. Medidas adoptadas por el centro de carácter organizativo.

\begin{tabular}{|c|c|c|c|c|}
\hline 0 & 1 & 2 & 3 & ¿? \\
\hline 0 & 1 & 2 & 3 & ¿'? \\
\hline 0 & 1 & 2 & 3 & ¿'? \\
\hline 0 & 1 & 2 & 3 & $\dot{c} ?$ \\
\hline 0 & 1 & 2 & 3 & ¿? \\
\hline 0 & 1 & 2 & 3 & ¿? \\
\hline 0 & 1 & 2 & 3 & ¿? \\
\hline
\end{tabular}

7.3. Medidas complementarias relativas a la satisfacción del personal del plan de convivencia. (datos objetivos)

O Nada, 1 Algo, 2 Bastante, 3 Mucho, ¿ं? No sabe, no contesta(escriba una X donde proceda)

35. Cumplimiento de objetivos previsto en el plan de convivencia escolar.

36. Grado de consecución de las competencias necesarias para alcanzar los

objetivos previstos por el centro en su plan de convivencia escolar.

37. Grado de aprovechamiento de las propuestas realizadas para su inclusión en el plan de convivencia.

38. Participación del personal en programas y actividades de mejora del plan

39. Participación del personal en actividades de formación y desarrollo profesional en relación a la convivencia escolar.

40. Participación del personal en los objetivos y fines del plan de convivencia.

41. Grado de eficacia en la transmisión de la información.

42. Grado de satisfacción obtenida en el tratamiento de las quejas.

\section{IMPACTO EN LA SOCIEDAD.}

Valore aquí los resultados del plan de convivencia en el entorno.

8.1. Grado de satisfacción respecto a los efectos en la comunidad.

0 nada, 1 Algo, 2 Bastante, 3 Mucho, ¿ं? No sabe, no contesta (escriba una $X$ donde proceda)

43. Comportamiento de los alumnos en el exterior.

44. Actividades conjuntas con otros centros educativos e instituciones.

45. Programas y actividades del plan que implican al entorno (escuelas de padres, grupos de mediación, actividades extraescolares, etc.)

46. Utilización de las instalaciones del centro para fines sociales y culturales del entorno.

47.

Campañas de información y sensibilización.

\begin{tabular}{|c|c|c|c|c|}
\hline 0 & 1 & 2 & 3 & ¿? \\
\hline 0 & 1 & 2 & 3 & ¿'? \\
\hline 0 & 1 & 2 & 3 & ¿'? \\
\hline 0 & 1 & 2 & 3 & ¿? \\
\hline 0 & 1 & 2 & 3 & $¿ ?$ \\
\hline 0 & 1 & 2 & 3 & ¿'? \\
\hline 0 & 1 & 2 & 3 & ¿'? \\
\hline 0 & 1 & 2 & 3 & ¿? \\
\hline
\end{tabular}

\section{RESULTADOS DEL PLAN DE CONVIVENCIA. \\ Valore aquí los efectos del plan de convivencia en el centro.}

9.1. La puesta en práctica del plan de convivencia ha contribuido a:

o Nada, 1 Algo, 2 Bastante, 3 Mucho, ¿'? No sabe, no contesta (escriba una $X$ donde proceda)

48. Reducir los actos de disrupción en el aula.

49. La colaboración entre el alumnado en las actividades de clase.

50. Reducir las faltas injustificadas de puntualidad.

51. Reducir las faltas injustificadas de asistencia a clase.

52. Reducir las faltas de respeto hacia los otros miembros de la comunidad educativa.

53. Reducir los daños en las instalaciones, recursos materiales o documentos del centro.

54. Reducir los daños en las pertenencias de los miembros de la comunidad educativa.

55. Reducir los casos de maltrato

\begin{tabular}{|c|c|c|c|c|}
\hline 0 & 1 & 2 & 3 & ¿? \\
\hline 0 & 1 & 2 & 3 & $\dot{d ?}$ \\
\hline 0 & 1 & 2 & 3 & $\dot{\imath} ?$ \\
\hline 0 & 1 & 2 & 3 & $\dot{c} ?$ \\
\hline 0 & 1 & 2 & 3 & $\dot{¿} ?$ \\
\hline 0 & 1 & 2 & 3 & $\dot{d ?}$ \\
\hline 0 & 1 & 2 & 3 & $\dot{c} ?$ \\
\hline 0 & 1 & 2 & 3 & ¿? \\
\hline
\end{tabular}


Análisis integral de la gestión de la convivencia escolar. Una propuesta de evaluación

56. Reducir el deterioro grave de las instalaciones, recursos materiales del Centro.

57. Mejorar las relaciones profesor-alumno

58. Mejorar las relaciones profesor-profesor

59. Mejorar las relaciones profesor-familia

60. Mejorar las relaciones alumno-alumno

61. Favorecer las relaciones entre grupos culturales

\begin{tabular}{|c|c|c|c|c|}
\hline 0 & 1 & 2 & 3 & ¿? \\
\hline 0 & 1 & 2 & 3 & $\dot{d} ?$ \\
\hline 0 & 1 & 2 & 3 & ¿? \\
\hline 0 & 1 & 2 & 3 & ¿? \\
\hline 0 & 1 & 2 & 3 & $\dot{d} ?$ \\
\hline 0 & 1 & 2 & 3 & ¿? \\
\hline
\end{tabular}

